\title{
Combination of Antibiotic, Efflux Pump Inhibitor and Autolysis Inhibitor Protects Mice from S. aureus Peritonitis by Inhibiting a-hemolysin-induced MAPKs/NF-KB/NLRP3 Activation
}

\author{
Wenjing Luan \\ Jilin University \\ Xiaolei Liu \\ Jilin University \\ Xuefei Wang \\ Jilin University \\ Yanan An \\ Jilin University \\ Yang Wang \\ Jilin University \\ Chao Wang \\ Jilin University \\ Lihui Ni \\ Jilin University \\ Fangxue $\mathrm{Ma}$ \\ Jilin University \\ Hong Zhou \\ Jilin University \\ Mingyuan Liu \\ Jilin University \\ Lu Yu ( $\nabla$ yu_lu@jlu.edu.cn ) \\ Jilin University https://orcid.org/0000-0002-5216-3843
}

\section{Research}

Keywords: Staphylococcus aureus (S. aureus), mastitis, cloxacillin (CXN), tetracycline (TC), thioridazine (TZ), combination therapy

Posted Date: December 10th, 2019 
DOI: https://doi.org/10.21203/rs.2.18440/v1

License: (c) (1) This work is licensed under a Creative Commons Attribution 4.0 International License. Read Full License 


\section{Abstract}

Background: The infection of staphylococcus aureus (S. aureus) is difficult to treat, our aim was to investigate the antibacterial abilities of a combination of antibiotic cloxacillin, efflux pump inhibitor thioridazine and autolysis inhibitor tetracycline and its anti-inflammation properties through inhibiting ahemolysin induced MAPKs/NF-KB/NLRP3 activation in vitro and in vivo.

Methods: The antibacterial susceptibility test and three-dimensional checkerboard method were utilized to investigate the synergistic antibacterial activity of the three-drug combination cloxacillin/thioridazine/tetracycline, a-hemolysin test and scanning electron microscope were used to assay the inhibition effects of the combination on the secretion of a-hemolysin and membrane-derived vesicles production from $\mathrm{S}$. aureus, Western blot and pharmacological inhibition assays were performed to test a-hemolysin -induced MAPKs/NF-KB/NLRP3 activation in macrophage in vitro.

Results: In vitro, the three-drug combination showed synergistic antistaphylococcal activity; the subinhibitory concentration of the combination significantly inhibited the secretion of a-hemolysin related to the number of vesicles produced by $\mathrm{S}$. aureus and significantly inhibited the expression of MAPKs/NF$\mathrm{KB} / \mathrm{NLRP3}$ proteins in the macrophages induced by $\mathrm{S}$. aureus a-hemolysin. In vivo, the drug combination significantly reduced bacterial colony-forming unit counts of the viscera in mouse peritonitis model of $\mathrm{S}$. aureus infection, the combination therapy reduced the primary inflammatory pathology and the release of bacterial-stimulated cytokines such as IL-1 $\beta$ and TNF- $\alpha$, and inhibited the expression of MAPKs/NF$\mathrm{KB} / \mathrm{NLRP} 3$ proteins in peritoneal macrophage.

Conclusions: Combination of antibiotic, efflux pump inhibitor and autolysis inhibitor owns good antistaphylococcal activity and significantly inhibit staphylococcal a-hemolysin and its inflammation, thus, the combination is a novel strategy of antibacterial and anti-inflammation.

\section{Introduction}

Staphylococcus aureus (S. aureus) infection is characterized by high complications, high recurrence rate, and strong drug resistance, and is the main cause of invasive infectious diseases(1). a-hemolysin (Hla), as the main virulence factor in the infection of $S$. aureus, can enhance the ability of $S$. aureus to destroy the host immune system and cell surface proteins(2). In addition, related studies have shown that Hla can be transported through $S$. aureus secretes membrane-derived vesicles (SMVs)(3), which is also the cause of Hla diffusion. In summary, to solve the $S$. aureus-caused diseases, research should be conducted on the proinflammatory pathogenicity of Hla and the production of vesicles.

Remarkably, S. aureus Hla have been reported could induce cell death through an NLRP3-dependent cellular necrosis program, resulting in the release of endogenous pro-inflammatory molecules(4). In addition, mitogen-activated protein kinases (MAPKs) are kinases involved in basic biological processes and expressing cellular responses to external stress signals, MAPKs (JNK, ERK1/2 and P38) regulate inflammatory and immune responses in macrophages(5). Moreover, activation of NF-KB pathway-related 
factors plays an important role in regulating inflammation(6). Thus, it is very important to make clear the possible sequence relation of MAPKs, NF-KB and NLRP3 in S. aureus Hla-induced inflamation and find its related inhibitors.

Combination therapy is an effective strategy to deal with refractory bacterial infection(7). Cloxacillin (CXN) is a beta-lactam drug that can be used to treat related infections caused by $S$. aureus (8). Thioridazine (TZ) is an efflux pump inhibitor that inhibits the bacterial efflux pump genes to some extent(9). Tetracycline (TC) is a broad-spectrum bacteriostatic agent and an autolysis inhibitor $(10,11)$. Therefore, we set out to develop a new type of three-drug prescription consist, who owns excelent antibacterial and anti-inflammation properties in vitro and in vivo, and its anti-inflammation may be mediated through inhibiting Hla-induced MAPKs/NF-KB/NLRP3 activation.

\section{Materials And Method}

\subsection{Ethics statement}

The mice were housed in isolation cages and given food and water in a timely manner. The ambient temperature is $24 \pm 1^{\circ} \mathrm{C}$ and the relative humidity is $40-80 \%$. All animal studies were conducted in accordance with experimental practices and standards approved by the Animal Welfare and Research Ethics Committee of Jilin University (No. IZ-2009-008).

\subsection{Cell and strain growth condition}

S. aureus 8325-4, DU1090 (isogenic Hla deletion mutant) was kept in the laboratory of the Institute of Veterinary Diseases, College of Veterinary Medicine, Jilin University. Macrophage RAW264.7, purchased from the Chinese Academy of Sciences Cell Bank (Shanghai, China). CXN, TZ and TC were purchased from Sigma-Aldrich and dissolved in sterile water at a concentration of $40,960 \mu \mathrm{g} / \mathrm{ml}$ under sterile conditions and stored at $-20^{\circ} \mathrm{C}$ until use.

\subsection{Antibacterial susceptibility test}

To determine the minimum inhibitory concentration (MIC) of 8325-4, DU1090 versus CXN, TZ and TC, a micro-broth dilution method was used according to the CLSI (formerly known as NCCLS) guidelines. Synergistic assays were performed in 96-well microtiter plates containing two antibacterial agents distributed along the transverse and longitudinal wells in a checkerboard pattern on a dioptric dilution basis. Each well contained $0.1 \mathrm{~mL}$ of a separate antimicrobial composition or broth control. Other conditions are the same as the MIC measurement conditions to determine the combined drug results. One drug concentration was fixed when evaluating three antibacterial drug combinations, and the other two drugs were operated as above. The in vitro graded inhibitory concentration index (FICl) of each group was calculated(12). The sum of the three drugs synergistically FICI ( $\Sigma F I C)$ was determined as follows: synergy $(\Sigma F I C \leq 1)$, additive effect $(\Sigma F I C=1)$, irrelevant effect $\left(\sum F I C=1-4\right)$, antagonism $(\Sigma F I C>4)(13)$.

\subsection{Hemolysis test}


S. aureus $8325-4 / D U 1090$ was incubated in tryptic soy broth (TSB) at $37^{\circ} \mathrm{C}$ to the exponential growth phase (OD $600 \mathrm{~nm}=2.5)$ with or without drug or drug combination. The culture was centrifuged and the supernatant was collected. $100 \mu \mathrm{l}$ containing different concentrations of supernatant were pre-incubated in Eppendorf tubes with defibrin rabbit red blood cells $(25 \mu \mathrm{L})$ and $875 \mu$ l hemolysis buffer $\left(20 \mathrm{mM} \mathrm{CaCl}_{2}\right.$, $0.125 \mathrm{M} \mathrm{NaCl}$ ) for $30 \mathrm{~min}$ at $37^{\circ} \mathrm{C}$. Hemolysis buffer was used as a negative control. After centrifugation, the supernatant was removed and the absorbance was measured at $450 \mathrm{~nm}$.

S. aureus strain 8325-4/DU1090 was incubated in tryptic soy broth (TSB) at $37^{\circ} \mathrm{C}$ to the exponential growth phase $\left(O D_{600 \mathrm{~nm}}=2.5\right)$ with or without drug combination. The culture was centrifuged at $8,500 \mathrm{~g}$ for $30 \mathrm{~min}$ at $4^{\circ} \mathrm{C}$, and the culture supernatant $(900 \mu \mathrm{L})$ was incubated with $100 \mu \mathrm{L}$ of LTCA for $12 \mathrm{~h}$ at $4^{\circ} \mathrm{C}$. The supernatant protein was collected and centrifuged at $8,500 \mathrm{~g}$ for $70 \mathrm{~min}$ at $4^{\circ} \mathrm{C}$. The extracted SMVs is then repeatedly frozen and thawed until broken. Protein Hla was tested by Western blot analysis. The primary antibody recognizing Hla was purchased from Sigma-Aldrich.

\subsection{Scanning electron microscope.}

Scanning electron microscopy (SEM) was used to analyze the number of spherical vesicles associated with the surface of $S$. aureus. Fixation of the cells should be fixed with $2.5 \%$ glutaraldehyde for 30 minutes at $-4^{\circ} \mathrm{C}$. Then, fixed with $1 \%$ osmium tetroxide at $-4^{\circ} \mathrm{C}$ for 30 min, dehydrated with a graded ethanol series, dried at a critical point, and covered with a gold film by sputtering. Floor. The sample was then analyzed by a scanning electron microscope.

\subsection{Vesicle extraction separation}

S. aureus 8325-4/DU1090 was incubated with drug-pretreated S. aureus 8325-4/DU1090. It was overnight at $37^{\circ} \mathrm{C}$ at $200 \mathrm{rpm}$ in an orbital shaker. The $S$. aureus strain was grown in $200 \mathrm{ml}$ of LB medium and grown to an optical density (OD $\left.{ }_{600}\right)$ of 2.0. Then, after a series of gradient centrifugation and filtration, the remaining supernatant was passed through a Beckman cryogenic ultracentrifuge (Beckman, USA) at $100,000 \mathrm{~g}$ for ultra-centrifugation at $4^{\circ} \mathrm{C}$ for $1 \mathrm{~h}$ to obtain membrane vesicles.

\subsection{Western blot analysis}

Bubble-treated RAW264.7 cells $\left(1 \times 10^{6}\right)$ obtained from S. aureus 8325-4/DU1090 (E:T ratio, 5:1) or pure $\mathrm{Hla}(100 \mu \mathrm{g} / \mathrm{ml}$, Sigma Aldrich) or ultracentrifuge). The drug combination (CXN: $0.015625 \mu \mathrm{g} / \mathrm{ml}, \mathrm{TZ}: 0.25$ $\mu \mathrm{g} / \mathrm{ml}, \mathrm{TC}: 0.03125 \mu \mathrm{g} / \mathrm{ml}$ ) was present or not at $37^{\circ} \mathrm{C}$ for $6 \mathrm{~h}$. Total protein was collected and the total protein concentration was determined by the BCA method. The same amount of protein was subjected to SDS-PAGE gel electrophoresis, and an ECL imaging system was used for image acquisition.

\subsection{Mouse $S$. aureus peritonitis model}

We used the previously described and widely used mouse peritonitis model to measure in vivo antibacterial effects $(14,15)$. In short, 6-week-old BALB/c female mice were inoculated with resuspended 
S. aureus (per $50 \mu \mathrm{L} 2 \times 10^{8}$ colony-forming unit (CFU). Each experimental group contained 30 mice. To further investigate the therapeutic effect of the drug combination in vivo, $100 \mu \mathrm{L}$ of drug or PBS was administered subcutaneously at 2 hours after infection with S. aureus 8325-4 / DU1090, and then administered at 12 hour intervals. After $72 \mathrm{~h}$, the infected mice were euthanized. In vivo, the concentration of the drug combination treatment group was: CXN $(1.6125 \mathrm{mg} / \mathrm{kg} / \mathrm{d}), \mathrm{TC}(3.125 \mathrm{mg} / \mathrm{kg} / \mathrm{d}), \mathrm{TZ}(25$ $\mathrm{mg} / \mathrm{kg} / \mathrm{d}$ ). Peritoneal macrophages were extracted and counted. Ascites colony counts of each group were performed. For histopathological analysis, formalin-fixed liver and spleen lung tissues were stained with hematoxylin and eosin and visualized using an Olympus BX53 fluorescence microscope with a $20 \times$ objective. Western blot analysis of protein extracted from peritoneal macrophages.

\subsection{Statistical analysis}

Comparisons of mean values from three experiments were statistically evaluated by analysis of variance, followed by One-Way ANOVA analysis. All statistical analyses were performed using SPSS software (version 11.5; SPSS).

\section{Results}

\subsection{Drug MIC and FICl measurement results}

To test the respective MICs of drug CXN, TZ and TC against $S$. aureus strains in vitro, we conducted a drug sensitivity test. The results showed that the MICs of CXN against $S$. aureus strain 8325-4 and DU1090 (an Hla-deleted strain) were $0.125 \mu \mathrm{g} / \mathrm{mL}$ and $0.125 \mu \mathrm{g} / \mathrm{mL}$ respectively; the MICs of TZ against strain 8325-4 and DU1090 were $16 \mu \mathrm{g} / \mathrm{mL}$ and $32 \mu \mathrm{g} / \mathrm{mL}$, respectively; the MICs of TC against strain $8325-4$ and DU1090 were $0.125 \mu \mathrm{g} / \mathrm{mL}$ and $0.125 \mu \mathrm{g} / \mathrm{mL}$, respectively (Table 1). To test the synergistic antibacterial activity of combination CXN, TZ and TC in vitro, we conducted a three-dimensional checkerboard method. The $\mathrm{FICl}$ results of the drug combination showed the two-drug combination of a certain concentration of CXN and TC, CXN and TZ, TZ and TC showed an additive effect on strain 8325-4, DU1090 (Table 2). Whereas the three-drug combination of a certain concentration of CXN, TZ and TC showed synergistic effect on strain 8325-4, and DU1090 (Table 2), for their sum FICl is less than 1.0 [19, ${ }^{20]}$, among them, we chose the synergistic three-drug combination of $\mathrm{MIC}_{\mathrm{CXN}}: \mathrm{MIC}_{\mathrm{TZ}}: \mathrm{MIC}_{\mathrm{TC}}=0.03125$ $\mu \mathrm{g} / \mathrm{mL}: 0.0625 \mu \mathrm{g} / \mathrm{mL}: 0.5 \mu \mathrm{g} / \mathrm{mL}$ as a candidate for further screen of three-drug prescription having simultaneous antibacterial and hemolysis-inhibiting activities.

\subsection{Subinhibitory concentration of threedrug combination inhibits hemolytic activity of Hla in vitro}

To test the effect of drug combination on $S$. aureus Hla production, but not inhibiting the bacterial growth, we selected a subinhibitory concentration of three-drug combination (1/2 MIC ${ }_{\mathrm{CXN}}: 1 / 2 \mathrm{MIC}_{\mathrm{TZ}}: 1 / 2 \mathrm{MIC}_{\mathrm{TC}}$ $=0.015625 \mu \mathrm{g} / \mathrm{mL}: 0.03125 \mu \mathrm{g} / \mathrm{mL}: 0.25 \mu \mathrm{g} / \mathrm{mL}$ ) for further experiments including Western blotting, hemolytic activity, and so on. Western blot analysis showed that CXN, TZ or TC alone at the concentration of subinhibitory three-drug combination inhibited the expression of Hla of $S$. aureus strain 8325-4 strain 
to varying degrees, the subinhibitory concentration of three-drug combination of $\mathrm{CXN}, \mathrm{TZ}$ and TC significantly inhibited the expression of Hla (Fig. 1A - B). Further blood plate experiment also verified that subinhibitory concentration of three-drug combination that of CXN, TZ and TC had best hemolysisinhibiting activities (Fig. 1E). Moreover, By measuring the absorbance of the supernatant at $450 \mathrm{~nm}$ with a spectrophotometer (Fig. 1C - D), we found that CXN, TZ or TC alone had a certain of hemolysisinhibiting activities, we also found subinhibitory concentration of three-drug combination had best hemolysis-inhibiting effect.

\subsection{Subinhibitory concentration of three-drug combination inhibits the production of SMVs}

Recent research evidence indicates that $S$. aureus secretes Hla-containing SMVs to the outside of the cell, and Hla is closely related to SMVs. In our study, we observed $S$. aureus strain 8325-4 produced much more SMVs on the surface than that of strain DU1090, wheras the drug CXN, TZ or TC alone inhibited the production of SMVs to varying degrees, especially, the subinhibitory concentration of three-drug combination treatment significantly inhibited the production of vesicles in strain 8325-4 than that of drugs alone treatment (Fig. 2A, 2D). In addition, we compared the expression of Hla in the SMVs extracted from strain 8325-4 treated by different drug treatments, the results showed that Hla was expressed in the SMVs isolated from strain 8325-4, and the subinhibitory concentration of three-drug combination significantly inhibited the expression of Hla, however, the expression of Hla in SMVs was not found in strain DU1090 (Fig. 2B - C). These results suggest that Hla is associated with SMVs, and that the subinhibitory concentration of three-drug combination suppresses the production of SMVs and the expression of Hla mediated by SMVs.

\subsection{Subinhibitory concentration of three-drug combination inhibits MAPKs, NF-KB and NLRP3 pathways in macrophage induced by $S$. aureus, SMVs or Hla}

In order to detect the effects of the three-drug combinations on the inflammatory pathway in S. aureus, Hla or vesicle-induced RAW264.7 cells, we performed Western blot analysis on the expression of MAPKs (Fig. 3A), NF-KB (Fig. 3B) and NLRP3 (Fig. 3C) related proteins in the macrophages. The results showed that the expression levels of p-JNK, p-ERK1/2, p-P38, NF-KB, IKK, ASC, IL-18, IL-1 $\beta$, NLRP3 and caspase-1 were significantly increased in strain 8325-4, 8325-4 MVs, and Hla inducement group compared with that of the blank control group. However, the expression levels of p-JNK, p-ERK1/2, p-P38, NF-KB, IKK, ASC, IL18 , IL-1 $\beta$, NLRP3 and caspase-1 were significantly downregulated in the strain DU1090 inducement groups compared with that of strain 8325-4, 8325-4 MVs or Hla groups. After the subinhibitory concentration of three-drug combination treatment, the expression levels of $p$-JNK, p-ERK1/2, p-P38, NFKB, IKK, ASC, IL-18, IL-1 $\beta$, NLRP3 and caspase-1 were significantly reduced in strain 8325-4, 8325-4 MVs or Hla groups compared with that of no drug treatment. Noteworthily, the expression of IKB were significantly lower in the strain 8325-4, 8325-4 MVs and Hla inducement groups than that of the blank control group, the strain DU1090 inducement group, and three-drug combination treatment group. These results indicates that the subinhibitory concentration of three-drug combination of $\mathrm{CXN}, \mathrm{TZ}$ and TC can 
inhibit the inflammatory response of MAPKs, NF-KB and NLRP3 pathways induced by S. aureus, vesicle and $\mathrm{Hla}$ in the macrophages in vitro.

\subsection{MAPKs (P38 and ERK1/2) mediates Hla-stimulated activation of NF-KB and NLRP3 inflammasome in macrophage}

Further experiments were performed to examine the upstream and downstream relationship between the MAPKs, NF-KB and NLRP3 in the Hla-activated inflammation pathways, we tested the expression of each pathway proteins in macrophages treated with Hla under pretreatment using the P38 inhibitor SB 203580, the ERK1/2 inhibitor PD 98059 and the JNKs inhibitor SP 600125, the NF-KB inhibitor BAY 11-7082, and the NLRP3 inhibitor Ac-YVAD-cmk. First, for MAPKs pathway, p38 inhibitor SB 203580, ERK1/2 inhibitor PD98059 and JNK inhibitor SP 600125 inhibited the expression of p-p38, p-ERK and p-JRK, respectively, in addition, the JNK inhibitor SP600125 inhibited the expression of p-p38, p-ERK, and p-JRK, but the p38 inhibitor SP203580 could not inhibit the expression of p-ERK and p-JNK; the ERK1/2 inhibitor PD98059 could not inhibit the expression of $p$-JNK. These results indicate that JNK is located upstream of ERK1/2 and P38 and that P38 is located downstream of ERK1/2. Second, we found that p38 inhibitor SB203580 and ERK1/2 inhibitor PD 98059 instead JNK inhibitor SP600125 significantly inhibited the production of NF-KB, IKK, ASC, IL-18, IL-1 $\beta$, NLRP3 and caspase-1 (Fig. 4). These results indicate that $S$. aureus Hla activated MAPKs (P38, ERK1/2, and JNK), NF-KB and NLRP3 pathways, P38 MAPK and ERK1/2 MAPKs (ERK1/2 located upstream of P38), but not JNK MAPK, mediated NF-KB and NLRP3 pathways.

\subsection{Three-drug combination treatment protects mice from S. aureus peritonitis in vivo}

As shown above, three-drug combination of CXN, TZ and TC exhibited an excellent antistaphylococcal activity and anti-Hla-activated inflammation in vitro. Next, we investigated the effect of three-drug combination protecting mice from $S$. aureus peritonitis in vivo. Gross pathological images, the results showed that the lung tissue of $S$. aureus strain 8325-4-infected mice showed deep pink with bleeding points, spleen tissue of strain 8325-4 infected mice was swollen, whereas the correspondent inflammation caused by strain DU1090 is weaker than its parental strain 8325-4 (Fig. 5A), and the correspondent inflammation caused by strain DU1090 was less than that of strain 8325-4. Especially, after three-drug combination treatment, the pathological changes of lung tissue and spleen tissue by $S$. aureus 8325-4-infected are weakened. However, the overall pathological changes of liver tissue were not obvious whatever in mice infected by strain 8325-4, strain DU1090 or treated by three-drug combination. The $S$. aureus $\mathrm{CFU}$ counts in the ascites cell supernatant were taken, and it was found that after threedrug combination treatment, the $S$. aureus CFU counts were decreased in some extent at $72 \mathrm{~h}$ in the peritoneal cavity, however, $S$. aureus $\mathrm{CFU}$ counts was significantly reduced at $144 \mathrm{~h}$ (Fig. 5B). Peritoneal macrophage counts showed that the number of macrophages in strain 8325-4 / DU1090-infected mice was significantly higher than that of no infection group, and the increase was significantly decreased after three-drug combination treatment (Fig. 5C).

Next, we performed Western blot analysis on the extracted mouse peritoneal macrophages (Fig. 6A). The results showed that the three-drug combination significantly reduced the levels of p-JNK, p-ERK1/2, p- 
P38, NF-KB, IKK, ASC, IL-18, IL-1 $\beta$, NLRP3 and caspase-1 (Fig. 6B). Serum ELISA analysis showed that the expression of IL-1 $\beta$ and the expression of TNF- $\alpha$ in the serum of the 8325-4-infected group were higher than those in strain DU1090-infected group and the blank group at $72 \mathrm{~h}$ and at $144 \mathrm{~h}$, respectively, and the values of strain DU1090-infected group were higher than those in the blank group, however, the counterpart of the three-drug combination treatment were significantly decreased in strain 8325-4infected group and in strain DU1090-infected group (Fig. 6C - F). All these results indicate that three-drug combination of CXN, TZ and TC has a good protective effect on mouse pneumonia caused by $S$. aureus Hla in vivo.

\section{Discussion}

A number of studies have shown that combined treatment can not only improve the antibacterial effect, but also reduce the toxicity caused by excessive concentration of a single drug(16). In this study, a threedrug combination consist of CXN, TZ and TC, was used to develop an efficient antistaphylococcal prescription. The results of this study indicate that the combination of CXN, TZ and TC has a synergistic effect against $S$. aureus (Table 1,2), thus, this new three-drug combination CXN/TZ/TC can also open a new direction for the treatment of other bacterial diseases.

S. aureus produces potent hemolysins(17) and leukotoxins. S. aureus secretes membrane-derived vesicles (SMVs) that deliver virulence factors to host cells and induce cytopathology(18). Thus, the screen of anti-Hla drugs is of great importance for the elimination of bacterial toxicity. The results of this study show that the three-drug combination can effectively inhibit the expression of Hla at a lower concentration than the single drug, indicating that the three-drug combination can effectively reduce the risk of diseases caused by $S$. aureus Hla. The drug combination inhibits the hemolytic activity and bacterial protein expression levels of Hla at concentrations below the MIC (Fig. 1), all these results indicate that the three-drug combination used here inhibits the bacteria and simultaneously inhibits the expression of the bacterial virulence protein Hla.

Studies have shown that the expression of MAPKs/NF-KB/NLRP3 pathway protein activity is a potential target for the study of anti-inflammatory drugs(19-21). Until now, there were few studies reported the relationship between $S$. aureus Hla-induced inflammation and simultaneous activation of MAPKs NF-KB and NLRP3. The results of this study demonstrated that the three-drug combination can significantly reduce the phosphorylation of MAPKs protein (JNK ERK1/2 and P38) in S. aureus-infected macrophages. The three-drug combination can also significantly reduce NF-KB and NLPR3-related inflammatory proteins in $S$. aureus-infected macrophages. Our results indicate that JNK is located upstream of ERK1/2 and P38, and P38 is located downstream of ERK1/2. In addition, P38 and p-ERK1/2 are located upstream of NF-KB and NLRP3. Taken together, the results indicate that the drug combination inhibits MAPKsmediated NF-KB and NLRP3 pathways activated by $S$. aureus or SMVs, Hla infection. This result indicates that the three-drug combination can exert its anti-inflammatory effect through MAPKs, NF-KB and NLPR3 pathways. 
In the in vivo experiment, the therapeutic effect against mouse peritonitis models obtained by the threedrug combination is remarkable and the results of ELISA analysis of serum also showed a significant decrease of the inflammatory factors IL-1 $\beta$ expression and TNF- $a$ after three-drug combination treatment. All these results indicate that drug has a good protective effect against mouse peritonitis caused by $S$. aureus Hla in vivo.

\section{Declaration}

\section{Ethics approval and consent to participate}

The mice in this experiment live in miniature isolation cages and ensure an adequate, healthy diet and water. The laboratory temperature was maintained at $24 \pm 1^{\circ} \mathrm{C}$ and the relative humidity was maintained at $40 \sim 80 \%$. All animal experiment criteria were based on the criteria for laboratory animal welfare and animal approval by the Research Ethics Committee of Jilin University (No. IZ-2009-008). The experimental protocol was reviewed and approved by the committee. In order to minimize animal suffering, all animals were tested under isoflurane anesthesia.

\section{Consent for publication}

Not applicable.

\section{Availability of data and material}

Please contact author for data requests.

\section{Funding}

This work was supported by the National Key R\&D Program of China (2016YFD0501302, 2017YFD0502200), the National Nature Science Foundation of China (No. 31172364), the Fund for Science \& Technology Development of Jilin Province (20150101108JC), the Important National Science and Technology Specific Projects (2012ZX10003002), the Program for New Century Excellent Talents in University (NCET-09-0434), the Project of the Education Department of Jilin Province (No. 2016444), the Science, Technology and Innovation Commission of Shenzhen Municipality (No. JCYJ2016031100720906, JSGG20160301100442775).

\section{Author's contribution}

Lu Yu participated in research design.

Wenjing Luan and Xuefei Wang conducted experiments.

Chao Wang, Lihui Ni and Hong Zhou constructied mice model.

Yanan An and Fangxue Ma performed data analysis. 
Yang Wang made Figures.

Lu Yu and Wenjing Luan wrote or contributed to the writing of the manuscript.

Xiaolei Liu, Mingyuan Liu and Lu Yu guided all the aspects of the study.

\section{Acknowlegments}

We thank Professor Timothy from Ireland for providing us with the S. aureus DU1090 strain.

\section{Competing Interests}

The authors declare no conflicts of interests.

\section{Reference}

1. McCaig LF, McDonald LC, Mandal S, Jernigan DB. Staphylococcus aureus-associated skin and soft tissue infections in ambulatory care. Emerging infectious diseases. 2006;12(11):1715-23.

2. Kebaier C, Chamberland RR, Allen IC, Gao X, Broglie PM, Hall JD, et al. Staphylococcus aureus alphahemolysin mediates virulence in a murine model of severe pneumonia through activation of the NLRP3 inflammasome. The Journal of infectious diseases. 2012;205(5):807-17.

3. Kim J, Bin BH, Choi EJ, Lee HG, Lee TR, Cho EG. Staphylococcus aureus-derived extracellular vesicles induce monocyte recruitment by activating human dermal microvascular endothelial cells in vitro. Clinical and experimental allergy : journal of the British Society for Allergy and Clinical Immunology. 2019;49(1):68-81.

4. Craven RR, Gao X, Allen IC, Gris D, Bubeck Wardenburg J, McElvania-Tekippe E, et al. Staphylococcus aureus alpha-hemolysin activates the NLRP3-inflammasome in human and mouse monocytic cells. PloS one. 2009;4(10):e7446.

5. Bi CL, Wang H, Wang YJ, Sun J, Dong JS, Meng X, et al. Selenium inhibits Staphylococcus aureusinduced inflammation by suppressing the activation of the NF-kappaB and MAPK signalling pathways in RAW264.7 macrophages. European journal of pharmacology. 2016;780:159-65.

6. Opitz B, Schroder NW, Spreitzer I, Michelsen KS, Kirschning CJ, Hallatschek W, et al. Toll-like receptor2 mediates Treponema glycolipid and lipoteichoic acid-induced NF-kappaB translocation. The Journal of biological chemistry. 2001;276(25):22041-7.

7. Segatore B, Bellio P, Setacci D, Brisdelli F, Piovano M, Garbarino JA, et al. In vitro interaction of usnic acid in combination with antimicrobial agents against methicillin-resistant Staphylococcus aureus clinical isolates determined by $\mathrm{FICl}$ and DeltaE model methods. Phytomedicine : international journal of phytotherapy and phytopharmacology. 2012;19(3-4):341-7.

8. Burdet C, Loubet P, Le Moing V, Vindrios W, Esposito-Farese M, Linard M, et al. Efficacy of cloxacillin versus cefazolin for methicillin-susceptible Staphylococcus aureus bacteraemia (CloCeBa): study protocol for a randomised, controlled, non-inferiority trial. BMJ open. 2018;8(8):e023151. 
9. Pule CM, Sampson SL, Warren RM, Black PA, van Helden PD, Victor TC, et al. Efflux pump inhibitors: targeting mycobacterial efflux systems to enhance TB therapy. The Journal of antimicrobial chemotherapy. 2016;71(1):17-26.

10. Ledala N, Wilkinson BJ, Jayaswal RK. Effects of oxacillin and tetracycline on autolysis, autolysin processing and atl transcription in Staphylococcus aureus. International journal of antimicrobial agents. 2006;27(6):518-24 .

11. Shittu AO, Okon K, Adesida S, Oyedara O, Witte W, Strommenger B, et al. Antibiotic resistance and molecular epidemiology of Staphylococcus aureus in Nigeria. BMC microbiology. 2011;11:92.

12. Yoon J, Urban C, Terzian C, Mariano N, Rahal JJ. In vitro double and triple synergistic activities of Polymyxin B, imipenem, and rifampin against multidrug-resistant Acinetobacter baumannii. Antimicrobial agents and chemotherapy. 2004;48(3):753-7.

13. Guelfi KC, Tognim MC, Cardoso CL, Gales AC, Carrara-Marrone FE, Garcia LB. In vitro evaluation of the antimicrobial activity of meropenem in combination with polymyxin $B$ and gatifloxacin against Pseudomonas aeruginosa and Acinetobacter baumannii. Journal of chemotherapy. 2008;20(2):1805.

14. Pachon-lbanez ME, Ribes S, Dominguez MA, Fernandez R, Tubau F, Ariza J, et al. Efficacy of fosfomycin and its combination with linezolid, vancomycin and imipenem in an experimental peritonitis model caused by a Staphylococcus aureus strain with reduced susceptibility to vancomycin. European journal of clinical microbiology \& infectious diseases : official publication of the European Society of Clinical Microbiology. 2011;30(1):89-95.

15. Sandberg A, Lemaire S, Van Bambeke F, Tulkens PM, Hughes D, von Eiff C, et al. Intra- and extracellular activities of dicloxacillin and linezolid against a clinical Staphylococcus aureus strain with a small-colony-variant phenotype in an in vitro model of THP-1 macrophages and an in vivo mouse peritonitis model. Antimicrobial agents and chemotherapy. 2011;55(4):1443-52.

16. Hobman JL, Crossman LC. Bacterial antimicrobial metal ion resistance. Journal of medical microbiology. 2015;64(Pt 5):471-97.

17. Otto M. Staphylococcus aureus toxins. Current opinion in microbiology. 2014;17:32-7.

18. Jeon $\mathrm{H}$, Oh MH, Jun $\mathrm{SH}$, Kim SI, Choi CW, Kwon HI, et al. Variation among Staphylococcus aureus membrane vesicle proteomes affects cytotoxicity of host cells. Microbial pathogenesis. 2016;93:18593.

19. Jahan S, Kumar D, Chaturvedi S, Rashid M, Wahajuddin M, Khan YA, et al. Therapeutic Targeting of NLRP3 Inflammasomes by Natural Products and Pharmaceuticals: A Novel Mechanistic Approach for Inflammatory Diseases. Current medicinal chemistry. 2017;24(16):1645-70.

20. Zhao G, Jiang K, Wu H, Qiu C, Deng G, Peng X. Polydatin reduces Staphylococcus aureus lipoteichoic acid-induced injury by attenuating reactive oxygen species generation and TLR2-NFkappaB signalling. Journal of cellular and molecular medicine. 2017;21(11):2796-808.

21. Fang L, Wu HM, Ding PS, Liu RY. TLR2 mediates phagocytosis and autophagy through JNK signaling pathway in Staphylococcus aureus-stimulated RAW264.7 cells. Cellular signalling. 2014;26(4):806- 


\section{Tables}

Due to technical limitations, the tables are only available as a download in the supplemental files section. Figures

Figure 1. Subinhibitory concentration of three-drug combination inhibits the hemolytic activity of Hla in vitro.

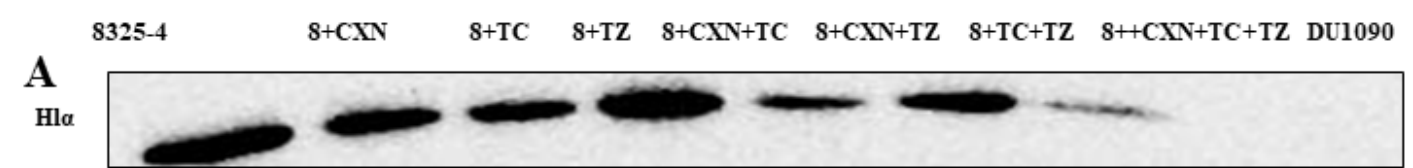

B

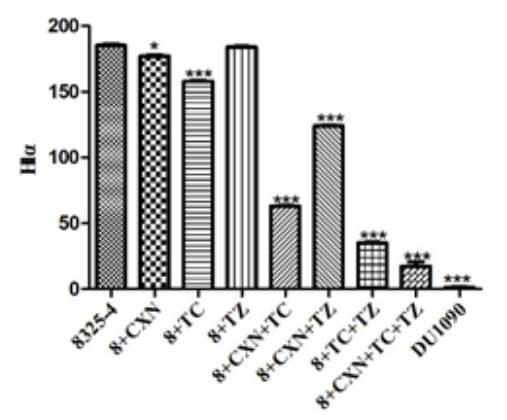

$8325-4$ $8+\mathrm{TC}$

$8+\mathrm{TZ} \quad 8+\mathrm{CXN}+\mathrm{TC} \quad 8+\mathrm{CXN}+\mathrm{TZ} \quad 8+\mathrm{TC}+\mathrm{TZ} \quad 8++\mathrm{CXN}+\mathrm{TC}+\mathrm{TZ} \quad \mathrm{DU} 1090$

C

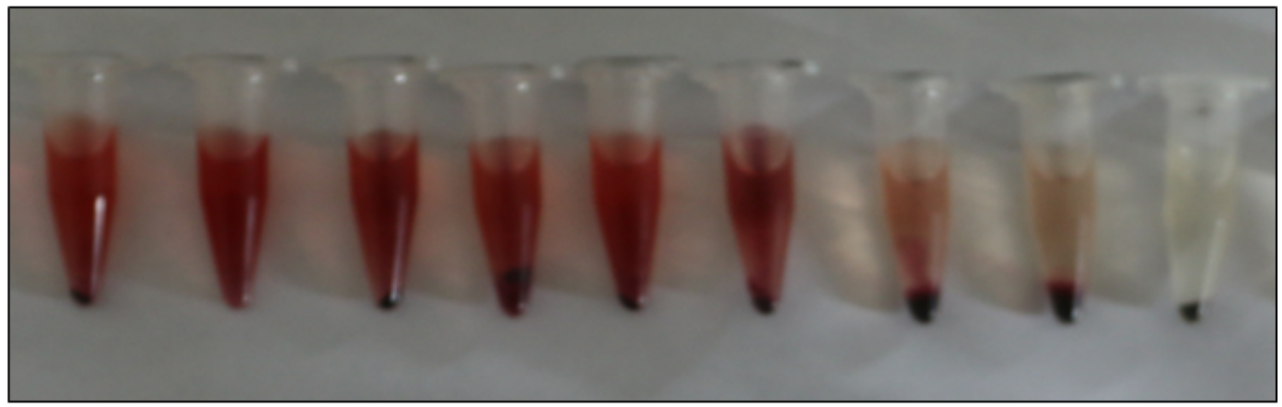

D

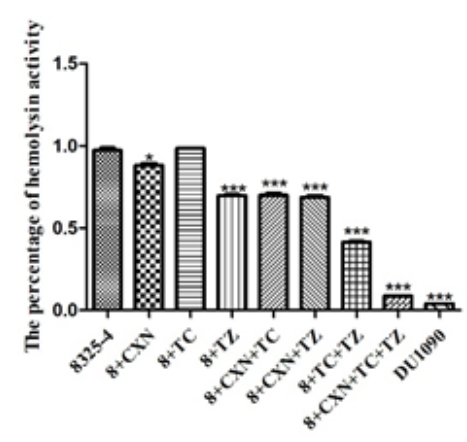

E

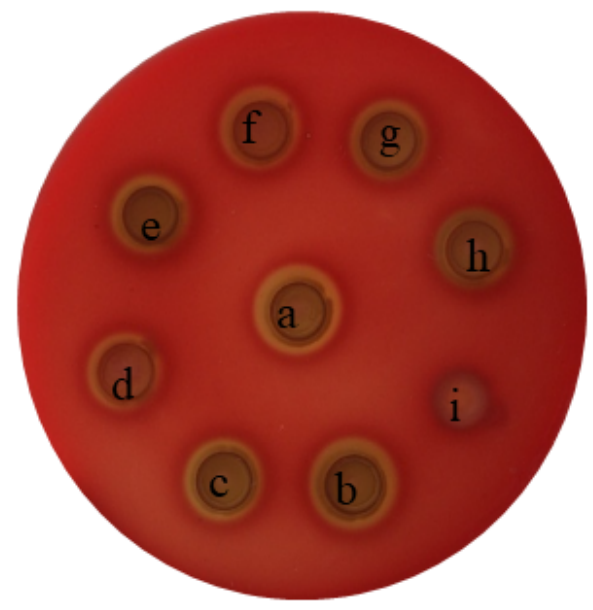




\section{Figure 1}

Subinhibitory concentration of three-drug combination inhibits the hemolytic activity of Hla in vitro. (A) Western blot analysis of expression of S. aureus strain 8325-4 in single drug and three drug combinations. (B) The expression of strain 8325-4 treated with different concentrations of drug and three drug combinations. (C) The hemolysis ability of the strain 8325-4 supernatant of each group of drugs was measured by measuring the absorbance at $450 \mathrm{~nm}$. (D) Percentage of hemolytic activity. (E) Hemolysis ability of strain $8325-4$ supernatant measured by rabbit co-cultured red blood cells. (a: 8325-4; b: 8325-4 + cloxacillin; c: 8325-4 + tetracycline; d: 8325-4 + thioridazine; e:8325-4 + cloxacillin + tetracycline; f: 8325-4 + cloxacillin + thioridazine; g: 8325-4 + tetracycline + thioridazine; h: 8325-4 + cloxacillin + tetracycline + thioridazine; i: DU1090). The data is the standard deviation of the means significantly different from strain $8325-4 .{ }^{*} P<0.05$, ${ }^{* *} p<0.01$, ${ }^{\star \star *} p<0.0001$. 
Figure 2. Subinhibitory concentration of three-drug inhibits the production of SMVs in $S$. aureus.

A $8325-4$

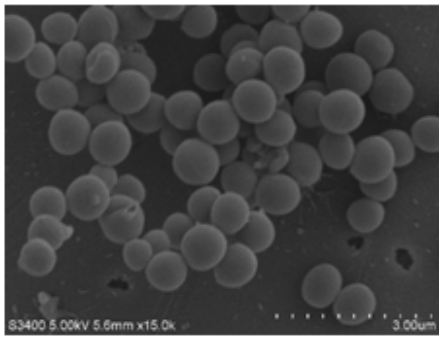

$8325-4+\mathrm{TZ}$

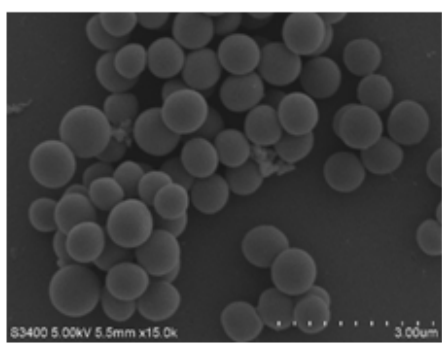

$8325-4+\mathrm{TC}+\mathrm{TZ}$

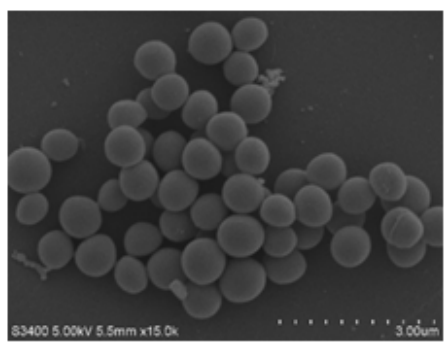

$8325-4+\mathrm{CXN}$

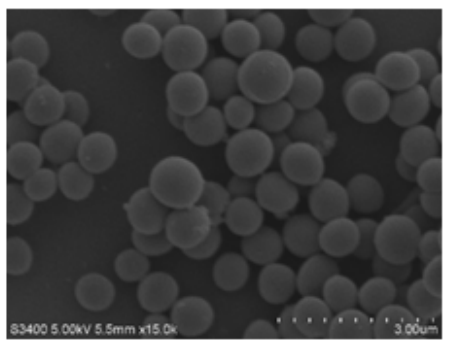

$8325-4+\mathrm{CXN}+\mathrm{TC}$

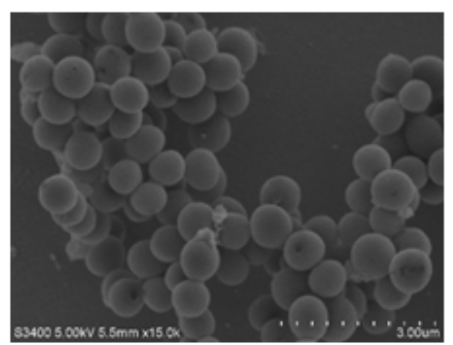

$8325-4+\mathrm{CXN}+\mathrm{TC}+\mathrm{TZ}$

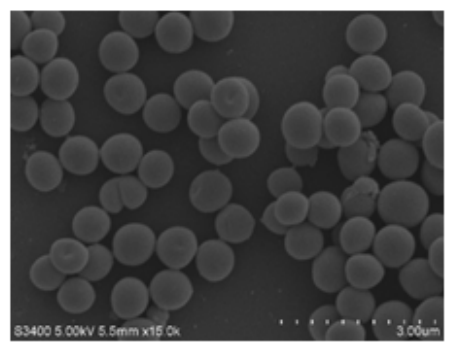

$8325-4+\mathrm{TC}$

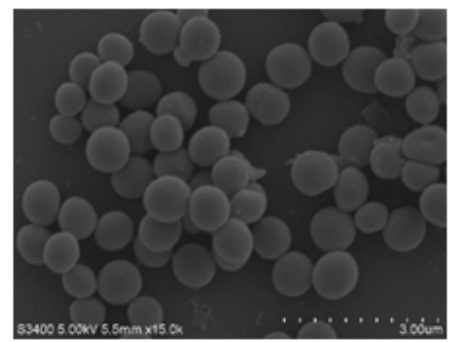

$8325-4+\mathrm{CXN}+\mathrm{TZ}$

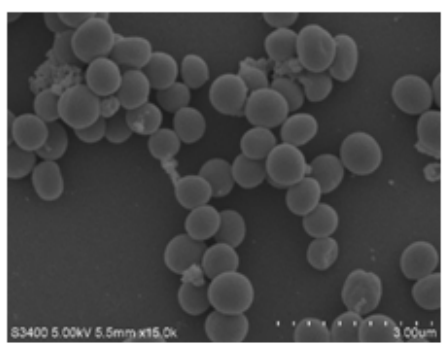

DU1090

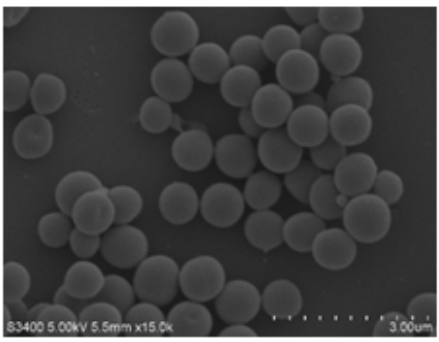

$\begin{array}{lllllllllll}\text { B } & 8325-4 & 8+\mathrm{CXN} & 8+\mathrm{TC} & 8+\mathrm{TZ} & 8+\mathrm{CXN}+\mathrm{TC} & 8+\mathrm{CXN}+\mathrm{TZ} & 8+\mathrm{TC}+\mathrm{TZ} & 8++\mathrm{CXN}+\mathrm{TC}+\mathrm{TZ} & \text { DUl1090 }\end{array}$ a-hla

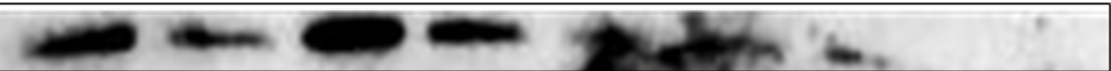

C

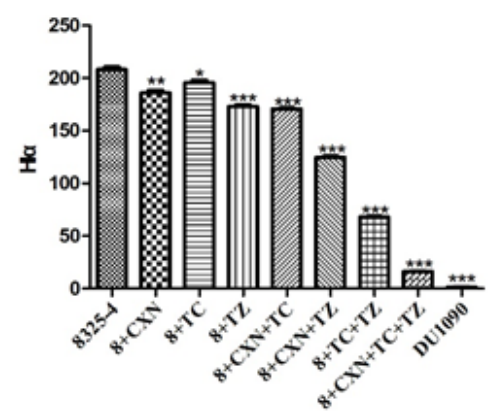

D

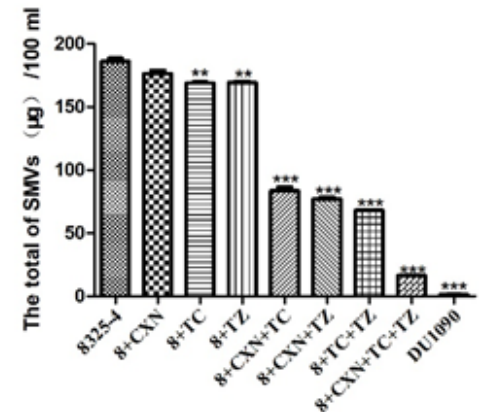

\section{Figure 2}

Subinhibitory concentration of three-drug inhibits the production of SMVs in S. aureus. (A) Scanning electron microscope for observation of drug treatment or untreated SMVs yield (15K $\times$ ). (B) The content of Hla was detected using a Western blot. (C) The content of Hla of S. aureus strain 8325-4 treated with different concentrations of drug and three drug combinations. (D) SMVs was extracted and quantified. 
The data is the standard deviation of the means significantly different from strain 8325-4. ${ }^{*} \mathrm{P}<0.05$, ${ }^{\star \star} \mathrm{p}$ $<0.01, * \star * p<0.0001$.

Figure 3. Subinhibitory concentration of three-Drug combination inhibits MAPKs, NF-кB, NLRP3 protein activation in $S$. aureus-, vesicular- or hla-induced macrophages.

A

B

C

8325-4 - + + - - CLO+TC+TZ- - + - $\mathrm{CXN}+\mathrm{TC}+\mathrm{TZ}--+$

DU1090 - - + + 8325-4MVs - + +

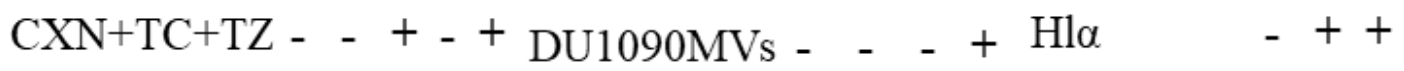

p-ERK

$\mathrm{p}-\mathrm{P} 38$

$\mathrm{p}-\mathrm{JNK}$
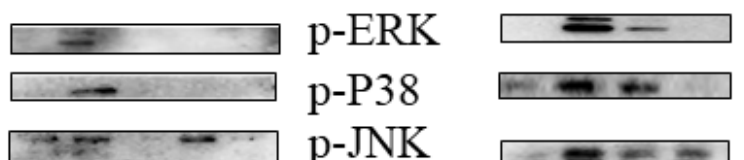

p-ERK

p-P38

$\mathrm{p}-\mathrm{JNK}$

$\mathrm{NF}-\mathrm{KB}$

NF-KB

IKK

$\mathrm{IKb}$

ASC

IL-18
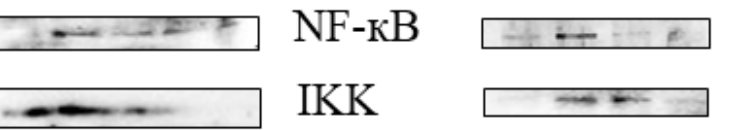

NF-кB

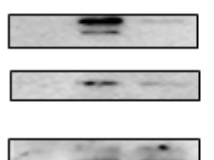

IL-1 $\beta$

NLRP3

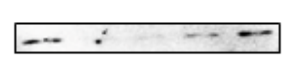

$\mathrm{IKb}$

$\ldots$ IKK

$-\infty \mathrm{IKb}$

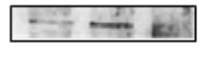

Caspase-1

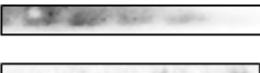

ASC

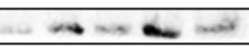

IL-18

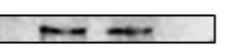

ASC

$=$ IL-18

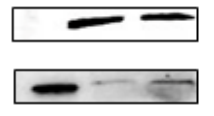

GAPDH

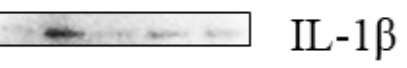

$\ldots \quad$ IL-1 $\beta$
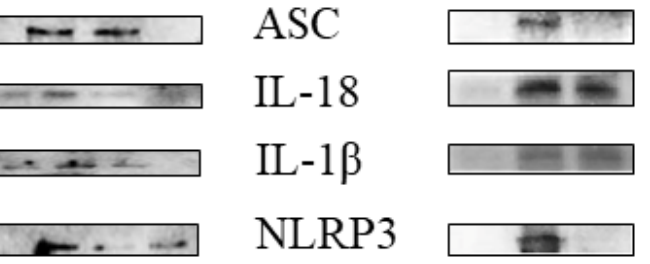

NLRP3

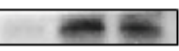

Nearer NLR3

1

Caspase-1
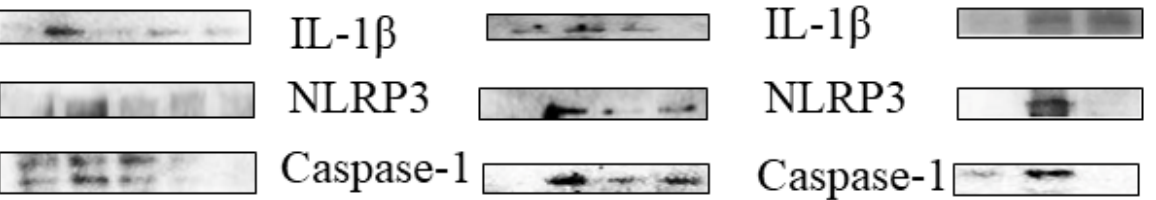

Caspase-1

$-\ldots$

GAPDH

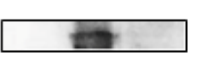

GAPDH

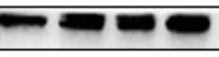

\section{GAPDH}

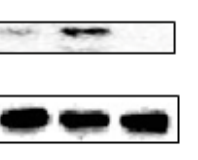

\section{Figure 3}

Subinhibitory concentration of three-Drug combination inhibits MAPKs, NF-KB, NLRP3 protein activation in S. aureus-, vesicular- or hla-induced macrophages. (A) Western blot was used to test the protein expression of MAPKs, NF-KB and NLRP3 in macrophages induced by S. aureus strain 8325-4 and 
DU1090 infection. (B) Western blot was used to test the protein expression of MAPKs, NF-KB and NLRP3 in the macrophages treated with the MVs of strain 8325-4 / DU1090 or by drug pretreatment. (C) Western blotting was used to analyze the expression of MAPKs, NF-KB and NLRP3 proteins in S. aureus hlainfected macrophages. The data is the standard deviation of the means significantly different from strain 8325-4. ${ }^{*} P<0.05, * * p<0.01, * * * p<0.0001$.

Figure 4. MAPKs mediates activation of NF-kB and NLRP3 inflammasome induced by Hl $\alpha$ in $М \Phi$.

\begin{tabular}{|c|c|}
\hline $\mathrm{Hl \alpha}$ & $-\quad++++++$ \\
\hline SP600125 & $-\quad-\quad+-\quad-$ \\
\hline PD98059 & $-\quad-\quad-\quad+--$ \\
\hline SB603580 & $-\quad+-$ \\
\hline BAY 11-7082 & $-\quad-\quad-\quad-\quad-\quad+$ \\
\hline Ac-YVAD-cmk & $-\quad-\quad-\quad+$ \\
\hline p-ERK & $c_{m}$ \\
\hline p-P38 & $\ldots-\ldots$ \\
\hline $\mathrm{p}-J \mathrm{NK}$ & $--m=-\infty$ \\
\hline $\mathrm{NF}-\mathrm{\kappa B}$ & ---- \\
\hline IKK & $r-\cdots$ \\
\hline IKB & $x=-$ \\
\hline $\mathrm{ASC}$ & $w=$ \\
\hline IL-18 & $\square-m=-m$ \\
\hline $\mathrm{IL}-1 \beta$ & 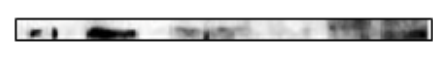 \\
\hline NLRP3 & 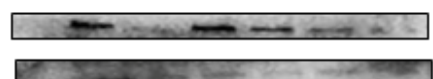 \\
\hline $\begin{array}{c}\text { Caspase-1 } \\
\text { GADPH }\end{array}$ & $\alpha-\infty-\infty-\infty$ \\
\hline
\end{tabular}

Figure 4 
MAPKs mediates activation of NF-KB and NLRP3 inflammasome induced by Hla in MФ. Upstream and downstream relationship between MAPKs, NF-KB and NLRP3 pathways.

\section{Figure 5. The corresponding indicators for in vivo drug combination treatment of mouse peritonitis.}

A

\section{K $\quad 8325-4 \quad 8325-4+\mathrm{CXN}+\mathrm{TC}+\mathrm{TZ} \quad \mathrm{DU} 1090 \quad \mathrm{DU} 1090+\mathrm{CXN}+\mathrm{TC}+\mathrm{TZ}$}
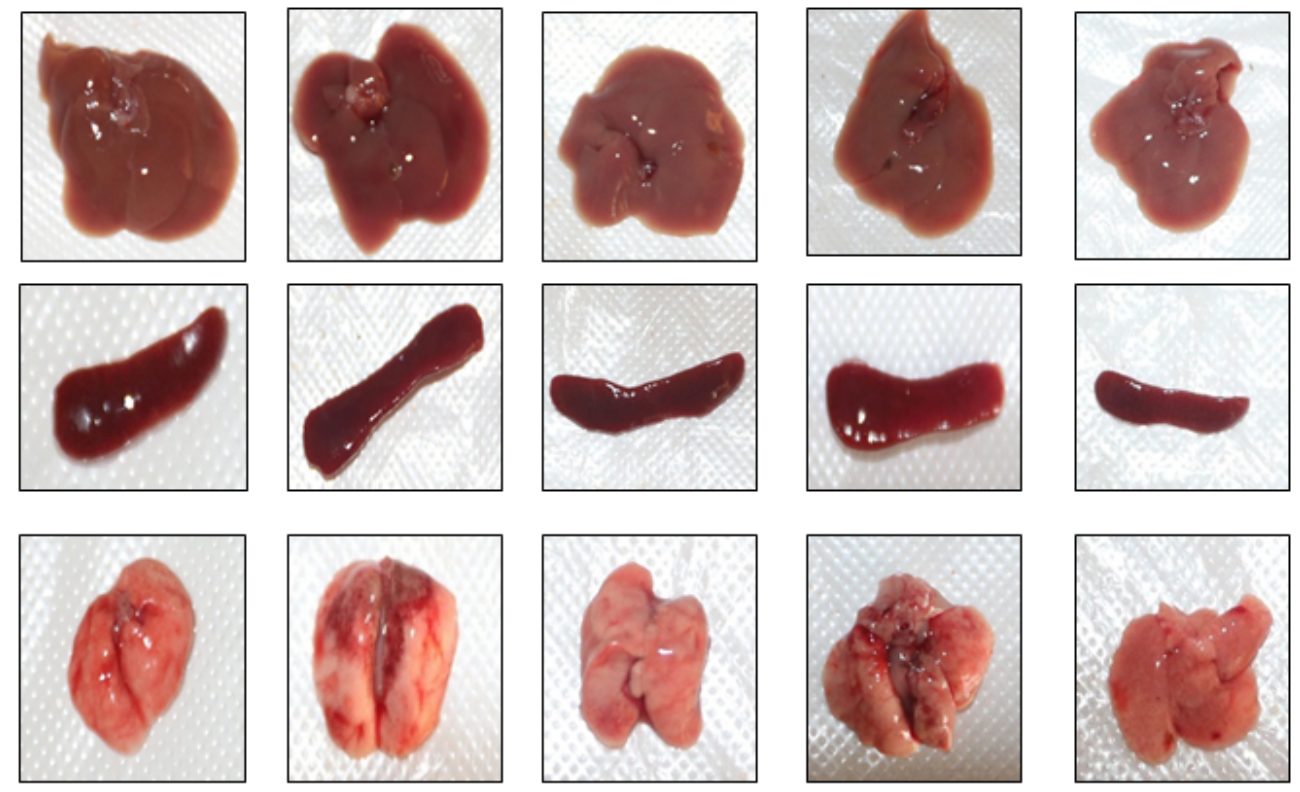

B

K $\quad 8325-4 \quad 8325-4+\mathrm{CXN}+\mathrm{TC}+\mathrm{TZ} \quad \mathrm{DU} 1090 \mathrm{DU} 1090+\mathrm{CXN}+\mathrm{TC}+\mathrm{TZ}$
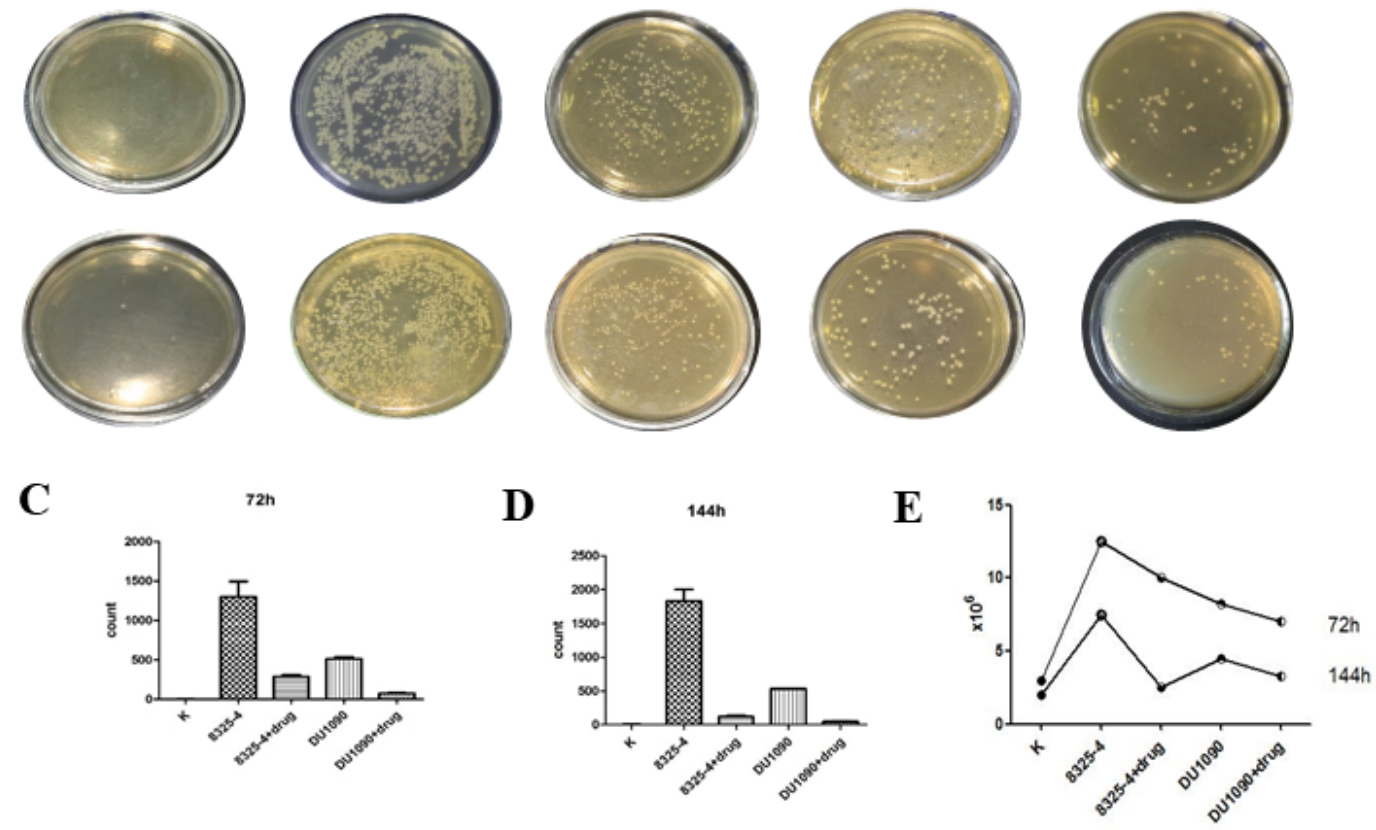

Figure 5

(A) Gross pathological images of liver, spleen and lung tissues. (B) Results of colony counts in ascites cell supernatants. (C) Results of colony counts in ascites cell supernatants at $72 \mathrm{~h}$. (D) Results of colony counts in ascites cell supernatants at $144 \mathrm{~h}$. (E) Peritoneal macrophage count results. 
Figure 6. Western blot and ELISA analysis on the extracted macrophages.

A

B

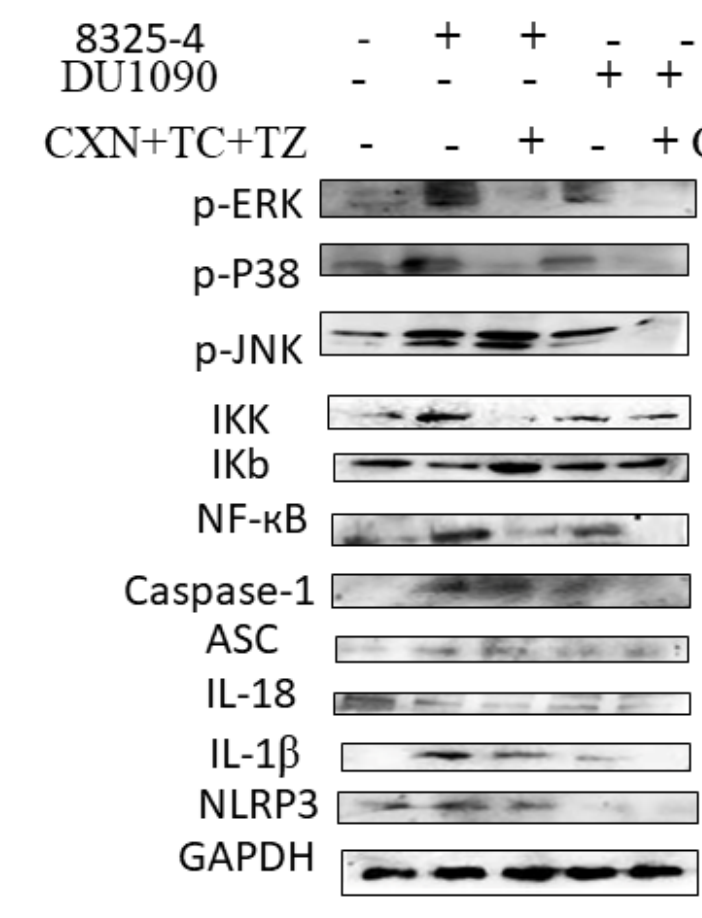

$\begin{array}{cccccc}8325-4 & - & + & + & - & - \\ \text { DU1090 } & - & - & - & + & +\end{array}$

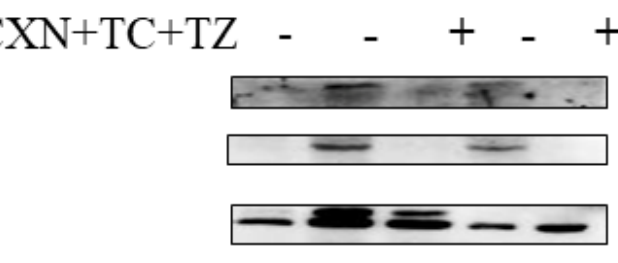

C
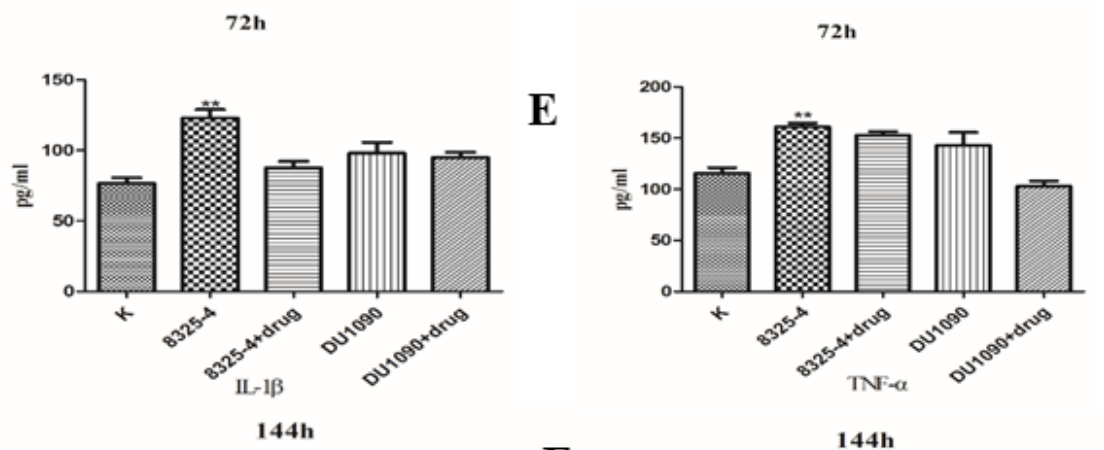

D
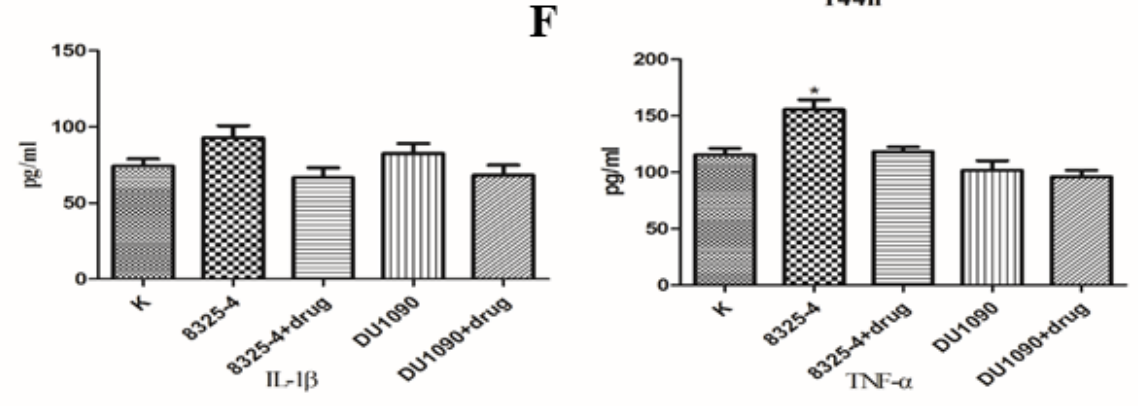

Figure 6

(A) Expression of peritoneal macrophage-associated inflammatory protein in the $72 \mathrm{~h}$ batch. (B) Expression of peritoneal macrophage-associated inflammatory protein in $144 \mathrm{~h}$ batch. (C) Expression of IL-1 $\beta$ in serum of the $72 \mathrm{~h}$ batch. (D) Expression of TNF- $a$ in serum of the $72 \mathrm{~h}$ batch. (E) Expression of IL$1 \beta$ in serum of the $144 \mathrm{~h}$ batch. $(\mathrm{F})$ Expression of TNF-a in serum of the $144 \mathrm{~h}$ batch. 


\section{Supplementary Files}

This is a list of supplementary files associated with this preprint. Click to download.

- Tables.PNG 